\title{
Bioresorbable scaffolds in the treatment of coronary artery disease
}

This article was published in the following Dove Press journal:

Medical Devices: Evidence and Research

II March 2013

Number of times this article has been viewed

\author{
Yaojun Zhang ${ }^{1,2}$ \\ Christos V Bourantas' \\ Vasim Farooq' \\ Takashi Muramatsu' \\ Roberto Diletti' \\ Yoshinobu Onuma' \\ Hector M Garcia-Garcia' \\ Patrick W Serruys' \\ 'Thoraxcenter, Erasmus Medical \\ Center, Rotterdam, The Netherlands; \\ ${ }^{2}$ Division of Cardiovascular Diseases, \\ Nanjing First Hospital, Nanjing \\ Medical University, Nanjing, People's \\ Republic of China
}

Correspondence: Patrick W Serruys Interventional Cardiology Department, Erasmus MC, 's-Gravendijkwal 230,

3015 CE Rotterdam, The Netherlands

Tel +3I 107035260

Fax +3I I0 4369I54

Email p.w.j.c.serruys@erasmusmc.nl

\begin{abstract}
Drug-eluting stents have reduced the risk of in-stent restenosis and have broadened the application in percutaneous coronary intervention in coronary artery disease. However, the concept of using a permanent metallic endovascular device to restore the patency of a stenotic artery has inherited pitfalls, namely the presence of a foreign body within the artery causing vascular inflammation, late complications such as restenosis and stent thrombosis, and impeding the restoration of the physiologic function of the stented segment. Bioresorbable scaffolds (BRS) were introduced to potentially overcome these limitations, as they provide temporary scaffolding and then disappear, liberating the treated vessel from its cage. Currently, several BRSs are available, undergoing evaluation either in clinical trials or in preclinical settings. The aim of this review is to present the new developments in BRS technology, describe the mechanisms involved in the resorption process, and discuss the potential future prospects of this innovative therapy.
\end{abstract}

Keywords: bioresorbable scaffold, drug-eluting stent, biodegradable, design, mechanism, coronary artery disease

\section{Introduction}

In 1977, Andreas Grüntzig introduced percutaneous transluminal coronary angioplasty, opening new horizons in the treatment of coronary artery disease (CAD).${ }^{1,2}$ Although the potential of this less invasive approach was apparent, the risk of acute occlusion and the high incidence of restenosis caused by elastic recoil and neointimal hyperplasia limited its application. ${ }^{3-5}$ Bare metal stents (BMSs) provided a partial solution to these problems, by eliminating the risk of acute occlusion and reducing the occurrence of restenosis. ${ }^{6-10}$ With the introduction of drug-eluting stents (DESs), the properties of the coated antiproliferative drug prolonged vessel wall healing, limited neointimal hyperplasia, and subsequently reduced the prevalence of target lesion revascularization (TLR) to less than $10 \%{ }^{11}$ This resulted in extensive application of DES, especially with the use of intravascular ultrasound for the patients with CAD. ${ }^{8,12-16}$ Conversely, delayed endothelization, and the hypersensitivity reaction of the vessel wall to the durable polymer, increased the risk of late and very late stent thrombosis (ST). ${ }^{17,18}$ Recent advances in DES technology with the use of either a bioresorbable or compatible polymer reduced the occurrence of ST but still failed to address other limitations of permanent metallic DES, namely the potential risk of neo-atherosclerosis, preclusion of surgical revascularization, and the distorted vessel wall physiology caused by the presence of a foreign body within the artery. ${ }^{15,19,20}$

For a number of decades, interventional cardiologists have pursued the possibility of a fully bioresorbable scaffold (BRS) that will initially safeguard the patency of 
the vessel, and then will disappear, thus allowing the artery to restore its physiological integrity. ${ }^{21-24}$ The feasibility of this concept was first investigated in the 1990s with the Igaki-Tamai ${ }^{\circledR}$ device (Igaki Medical Planning Co, Ltd, Kyoto, Japan); this process was however temporarily halted with the introduction of metallic DESs. Recently, BRS has been heralded as the fourth revolution, rendering a novel treatment termed vascular restoration therapy (VRT) in interventional cardiology. ${ }^{25}$ The clear feature of VRT is that vessel scaffolding is degraded evanescently, and therapeutic advantages may be derived from allowing natural vasomotor response in the vessel. In addition, the underlying benefits involve decreased or eliminated late ST, facilitated re-intervention due to the complete reabsorption of the implanted scaffold, improved noninvasive imaging of the target site using computed tomography or magnetic resonance during post-intervention follow-up, and shortened prescription of dual anti-platelet therapy. ${ }^{26}$ Accordingly, for $\mathrm{BRS}$, it is potentially a ideal therapy for CAD, although there is still a long way to go to make improvements.

Over the last 5-10 years, considerable efforts were made to develop new fully bioresorbable devices. Currently, BRS technology has been gradually matured, and there are numerous devices available which undergo preclinical or clinical testing. In this review article, we present the mechanisms of BRS technology, the evolution of individual devices, discuss the developments of the currently available BRS, and underline their potential value in the treatment of CAD.

\section{Design and resorption of the BRSs}

For a BRS to be as effective as the currently available DES, it should have an increased radial strength that should be maintained for at least 3-6 months post device implantation and incorporate an antiproliferative drug that would control neointimal formation and prevent restenosis. The prolonged radial strength can be provided by an appropriate scaffold design (eg, increased strut thickness, closed cell design) and by the incorporation of a resilient resorbable material (polymer or metallic alloy) which would gradually resorb so as to prevent late recoil and safeguard the patency of the vessel. Although the first generation BRS did not include an antiproliferative drug, more recent updated revisions incorporate a fully biodegradable polymer that controls the release of a drug elution with antiproliferative properties.

The first polymeric (nonbioresorbable polyethyleneterephthalate) braided mesh stent was designed in $1992 .{ }^{27}$ Preclinical evaluation in porcine models demonstrated that the developed polymeric stent had comparable safety and efficacy to the cotemporary metallic stent. In 1996, van der Giessen et al introduced the Wiktor stent, coated with five different fully bioresorbable polymers. ${ }^{28}$ The experimental study showed an excessive inflammatory reaction of the vessel wall to the device, resulting in increased neointimal proliferation and thrombus formation. One year later, Lincoff et al reported the first high molecular weight poly-Llactic acid (PLLA, $321 \mathrm{kDa}$ ) coated stent, that in contrast to the low molecular weight $(80 \mathrm{kDa})$ PLLA coated stent, appeared to be well tolerated by the vessel wall. ${ }^{29}$ Yamawaki et al were the first to develop a fully BRS, consisting of PLLA and a tyrosine kinase inhibitor. Evaluation of the device in porcine models demonstrated the safety and efficacy of the device and supported its implantation in humans in $1998 .^{30}$ These preliminary encouraging results attracted interest and drove researchers and industry towards the development of several BRSs with different bioresorbable properties and composition. ${ }^{19,31-33}$

Most of the currently available BRSs (eg, Igaki-Tamai scaffold, Absorb ${ }^{\text {TM }}$ [Abbott Vascular, Santa Clara, CA, USA] bioresorbable vascular scaffold [BVS]) are composed of PLLA. The catabolism of the PLLA incorporates five stages, with the resultant degradation of the polymer to carbon dioxide and water (Figure 1). ${ }^{34,35}$ The first phase includes hydration of the polymer, which starts to absorb water from the surrounding tissue. The absorbed water catalyzes a chain scission at an ester bond, resulting in the degradation of the polymer (second stage). Gradually the polymer loses its cohesive strength and fragments into segments with a lower molecular weight (third stage). Polymer chains become progressively hydrophilic through their hydrolysis to shorter lengths. The outcomes of the abovementioned process are hydrophilic monomers that can be phagocytized by macrophages (fourth phase). After phagocytosis, the soluble monomer (L-lactate) is catabolized to pyruvate and ultimately to carbon dioxide and water through the Krebs cycle (fifth stage). ${ }^{36,37}$

Tyrosine polycarbonate is another polymer used in BRS technology (REVA BRS). Its catabolism includes hydrolysis of the polymer to carbon dioxide and iodinated-desaminotyrosyltyrosine ethyl esters, which are further hydrolyzed to ethanol and iodinated-desaminotyrosyl-tyrosine. Cleavage of the latter results in tyrosine molecules and iodinated-desaminotyrosine, which are finally catabolized into carbon dioxide and water through the Krebs cycle (Figure 2).

Another material used in BRSs to date is magnesium, the only metal that has been implemented in BRS technology 


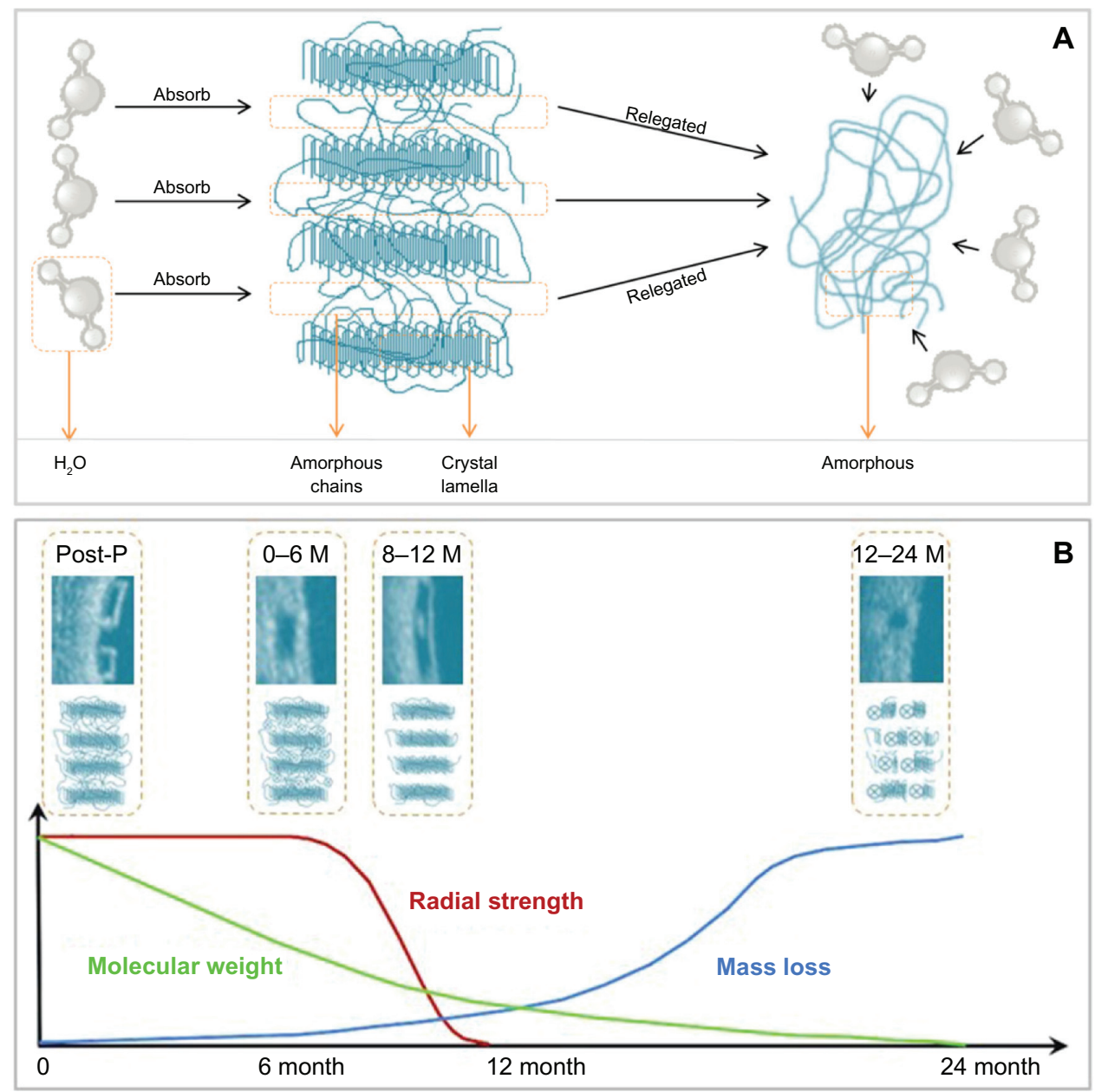

Figure I Schematic representation of the poly-L-lactic acid bioresorption process. (A) Crystal lamella, interconnected by amorphous chains, absorbs water from the surrounding tissue after implantation in the coronary lesion. The amorphous polymer is more susceptible to hydration than the semicrystalline polymer. (B) Schematic representation of changes in the radial strength, molecular weight, and mass of the scaffold with time.

Note: The optical coherence tomography and corresponding molecular images, illustrate the changes in strut appearance and composition of the scaffold with time.

Abbreviations: $M$, months; $P$, procedure.

(absorbable metallic stent [AMS]). A limitation of magnesium is its fragility; thus, it has been mixed with several elements such as zirconium, yttrium, and other rare earth metals to provide it with adequate radial strength. An advantage of the AMS is the fact that the degradation of the magnesium alloy to inorganic salts (Figure 3) triggers only a minor inflammatory response, and creates an electronegative charge that has been shown to have an anti-thrombogenic effect. ${ }^{38}$

\section{Currently available BRSs}

Currently, there are more than 14 BRSs available (Table 1 and Figure 4). Most are still either under preclinical evaluation or being examined in the clinical setting. Of note, two have already acquired certificate Conformité Européenne (CE) mark approval and are used in clinical practice: the Igaki-Tamai stent for the treatment of peripheral vascular disease, and the Absorb BVS for CAD. The following sections describe the BRSs that are under clinical or preclinical evaluation, and present the evidence from the first applications of BRSs in clinical settings or experimental studies.

\section{Igaki-Tamai scaffold}

The Igaki-Tamai scaffold was the first fully bioresorbable stent implanted in humans and was made of PLLA. The first revision of scaffold had a helical zigzag design, was mounted on a standard angioplasty balloon, and was both thermal self-expanding and balloon expandable. Scaffold expansion was performed with the use of heated contrast (up to $70^{\circ} \mathrm{C}$ ). The device continued expanding after its implantation over a 20-30 minute period, until it reached its final dimensions.

The first-in-man (FIM) study of the Igaki-Tamai scaffold included 15 patients treated with 25 scaffolds, and showed no major adverse cardiac event (MACE) at 


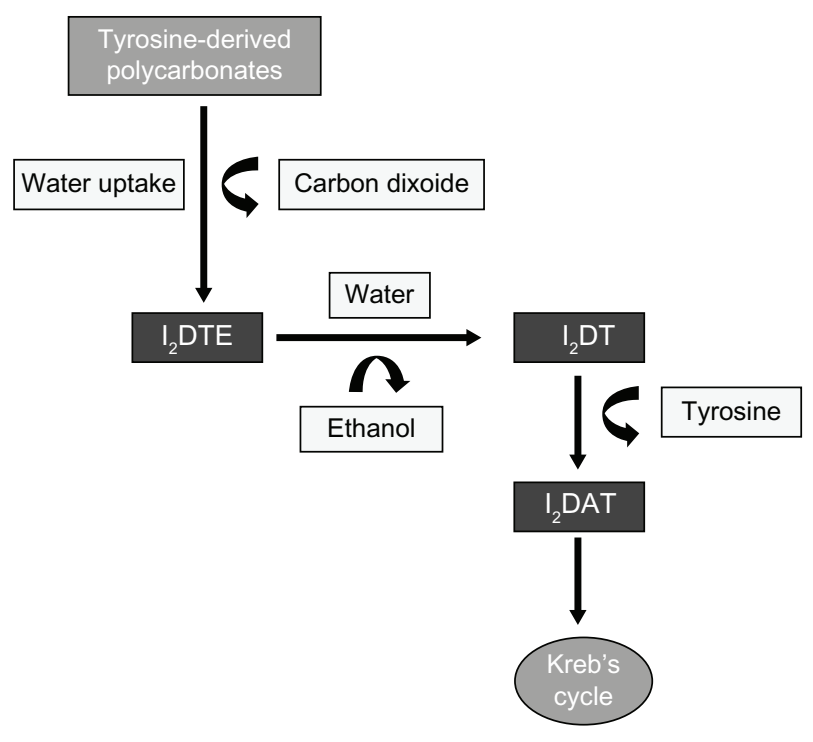

Figure $\mathbf{2}$ The metabolism of tyrosine-polycarbonate scaffold. Initial hydrolysis of the tyrosine-polycarbonate produces iodinated-desaminotyrosyl-tyrosine ethyl esters (I,DTE), and releases carbon dioxide.

Notes: $I_{2} \mathrm{DTE}$ is hydrolyzed to iodinated-desaminotyrosyl-tyrosine $\left(\mathrm{I}_{2} \mathrm{DT}\right)$ and ethanol. Cleavage of $\mathrm{I}_{2} \mathrm{DT}$ produces tyrosine, and iodinated-desaminotyrosine $\left(\mathrm{I}_{2} \mathrm{DAT}\right)$, which enters into the Kreb's cycle.

the first 30 days and only one TLR at 6-month follow-up. ${ }^{39}$ Angiographic follow-up performed at day 1 after the device implantation revealed a similar percentage diameter stenosis and minimal lumen diameter (MLD) compared with the post-procedural values $(12 \% \pm 8 \%$ versus $13 \% \pm 11 \%$; $2.59 \pm 0.35 \mathrm{~mm}$ versus $2.58 \pm 0.32 \mathrm{~mm}$ ). At 3 months, the MLD decreased $(1.88 \pm 0.59 \mathrm{~mm})$, with minimal progression thereafter at 6 months follow-up $(1.84 \pm 0.66 \mathrm{~mm})$. Serial intravascular ultrasound (IVUS) examinations demonstrated a gradual increase in the scaffold cross-sectional area (from $7.42 \pm 1.51 \mathrm{~mm}^{2}$ post stenting, to $8.18 \pm 2.42 \mathrm{~mm}^{2}$ at 3 months, and $8.13 \pm 2.52 \mathrm{~mm}^{2}$ at 6 months; $P<0.1$, $P<0.1$; respectively), whilst the luminal dimensions decreased at 3 months, and remained unchanged at 6 months (from $7.42 \pm 1.51 \mathrm{~mm}^{2}$ after stenting to $5.67 \pm 2.42 \mathrm{~mm}^{2}$ at 3 months, and $5.63 \pm 2.70 \mathrm{~mm}^{2}$ at 6 months; $P<0.005$, $P<0.001$; respectively).

Recently, Nishio et al reported the long-term follow-up ( $>10$ year) of an observational prospective study which enrolled 50 patients (63 lesions) implanted with IgakiTamai scaffolds (84 scaffolds). ${ }^{40,41}$ The 10 -year cumulative event-free survival rates of all-cause death, cardiac death, and MACE were $87 \%, 98 \%$, and $50 \%$, respectively, while the TLR rate was $16 \%$ at 1 year, $18 \%$ at 5 years, and $28 \%$ at 10 years. Angiographically, the late lumen loss (LLL) was $0.91 \pm 0.69 \mathrm{~mm}$ at 6 months, which improved to $0.67 \pm 0.45 \mathrm{~mm}$ at 1 year, and was maintained at 3 years follow-up $(0.59 \pm 0.50 \mathrm{~mm})$. IVUS examinations showed a reduction in the minimal lumen area at 6 months (from $5.44 \pm 1.89 \mathrm{~mm}^{2}$ after stenting to $3.64 \pm 1.68 \mathrm{~mm}^{2}$ ), which started increasing at $1\left(4.06 \pm 1.61 \mathrm{~mm}^{2}\right)$ and $3\left(5.18 \pm 2.09 \mathrm{~mm}^{2}\right)$ years. IVUS echogenicity revealed raised hyper-echogenicity of the scaffolded segment post device implantation, and then subsequently started decreasing to reach the pre-implantation values at 3 years follow-up.

Although the short- and long-term follow-up results were encouraging, this device failed to progress, mainly due to the large guide catheters $(8 \mathrm{~F})$ required for implantation, and the concerns that the heated contrast used for device deployment may cause vessel wall injury. The new generation Igaki-Tamai scaffold potentially overcomes these pitfalls, as it can be implanted through a $6 \mathrm{~F}$ guide catheter without the need for

\section{Device functionality of DREAMS}

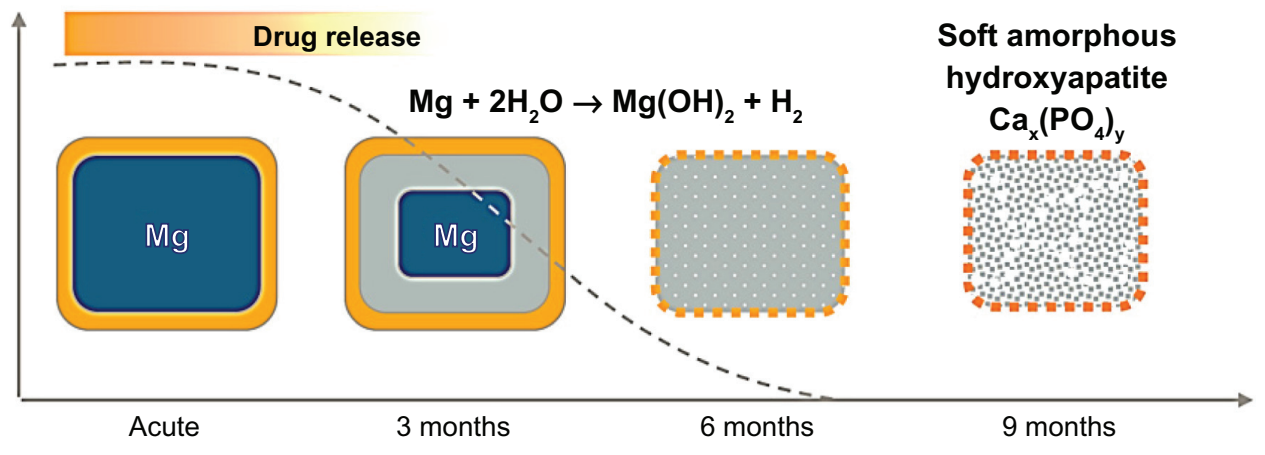

Figure 3 Schematic representation of the resorption process in the drug-eluting absorbable magnesium scaffold.

Notes: The release of the anti-proliferative drug occurs within the first 3 months after device implantation. Hydrolysis of the scaffold affects the radial strength of the scaffold, resulting in a gradual degradation of the device into a soft amorphous hydroxyapatite at 9 months follow-up.

Abbreviation: DREAM, drug-eluting absorbable metallic stent. 


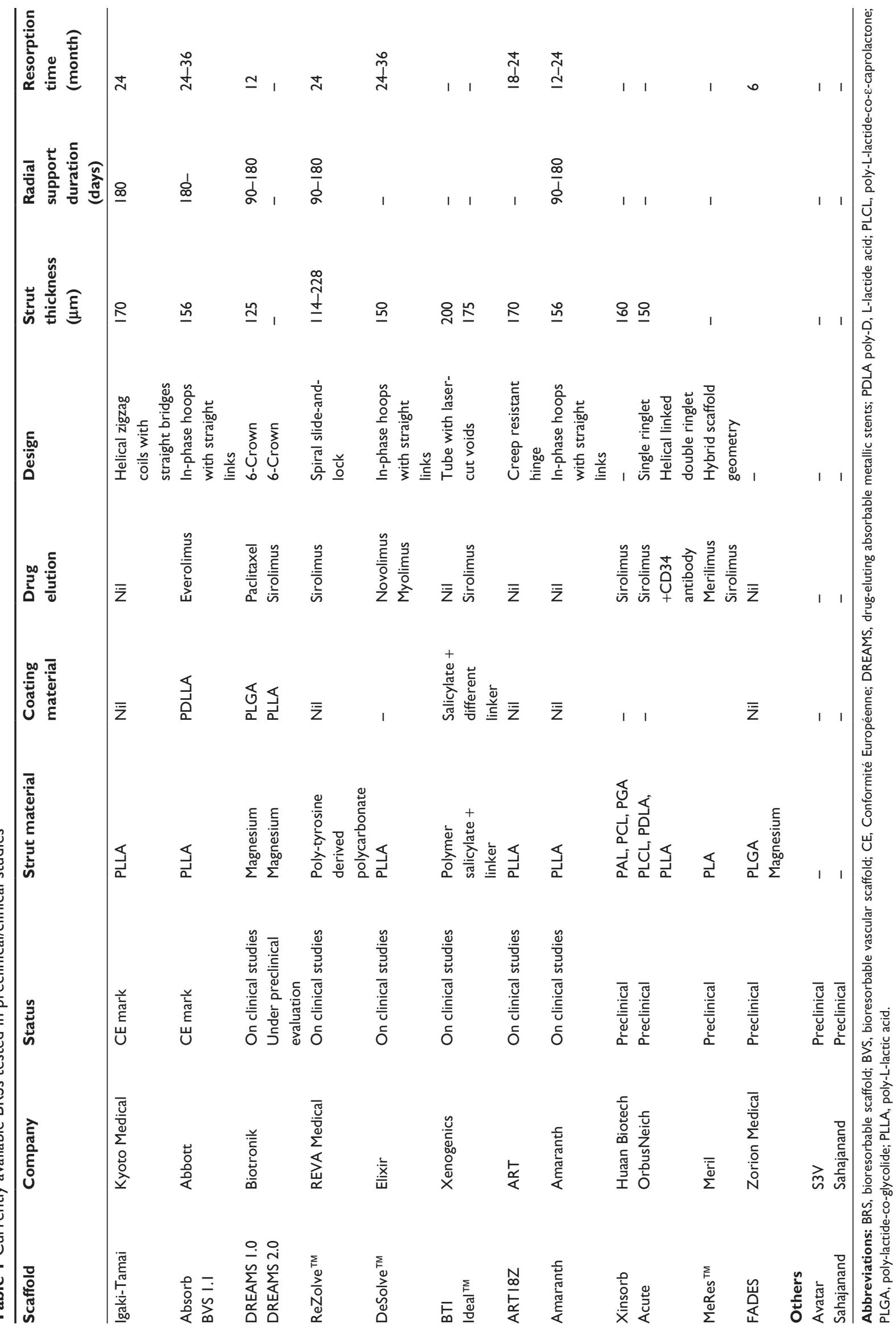




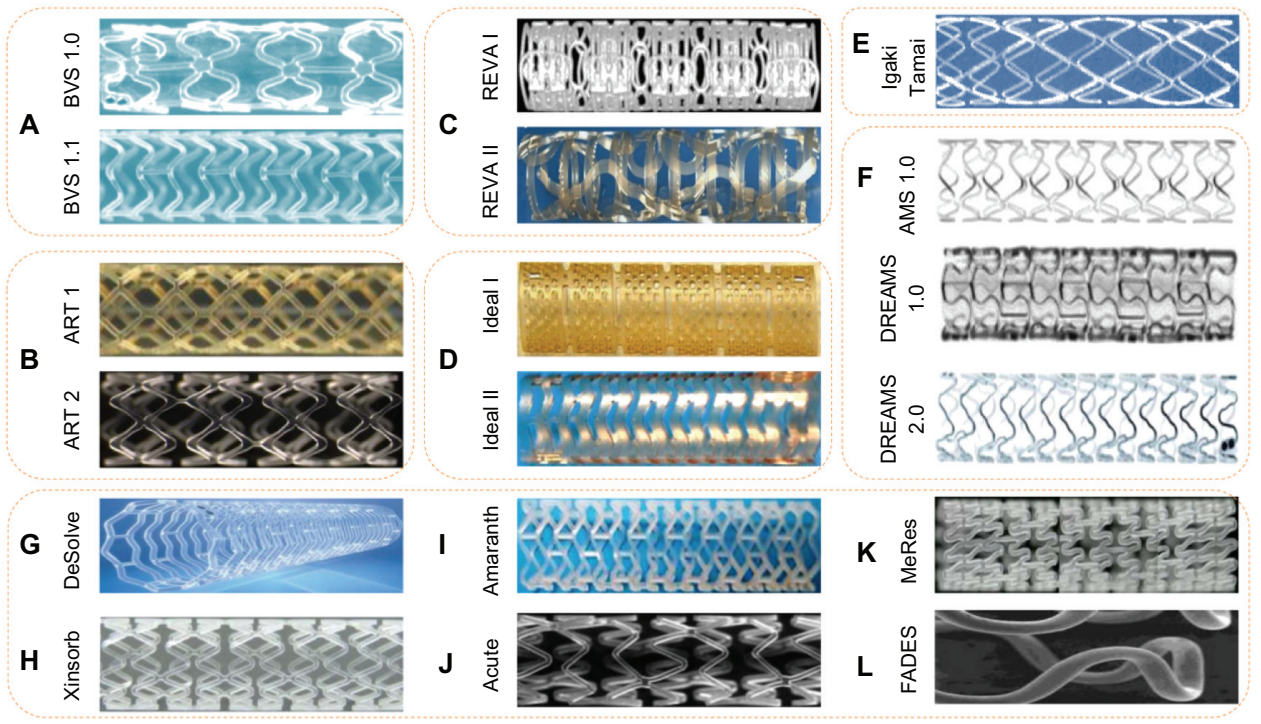

Figure 4 Currently available BRSs. (A) Abbott Vascular BRS I.0, I.I; (B) ART BRS I, 2; (C) REVA Medical BRS I, II; (D) IDEAL scaffold I, II; (E) Igaki-Tamai scaffold; (F) magnesium absorbable stents; (G) DeSolve ${ }^{T M}$ BRS; (H) Xinsorb BRS; (I) Amaranth BRS; (J) Acute BRS; (K) MeRes BRS; and (L) FADES BRS. Abbreviations: BRS, bioresorbable scaffold; DREAMS, drug-eluting absorbable metallic stents.

a heated contrast agent. The device is currently undergoing preclinical evaluation in Germany.

\section{Absorb BVS}

The Absorb BVS has a backbone composed by PLLA, and is coated with a poly-D,L-lactide (PDLA) polymer that contains the anti-proliferative drug everolimus. Histology based studies in porcine models have demonstrated that scaffold resorption is completed within 3 years post device implantation (Figure 5).42

The first generation of Absorb BVS (BVS 1.0) was examined in the ABSORB Cohort A Trial (A Clinical Evaluation of the Bioresorbable Everolimus Eluting Coronary Stent System in the Treatment of Patients with de Novo Native Coronary Lesions). The trial aimed to assess the feasibility and safety of the scaffold in patients with single de novo coronary artery lesion. In this prospective, open-label study, 30 patients who had stable, unstable, or silent ischemia were enrolled. ${ }^{33}$ The composite endpoint of MACE was cardiac death, myocardial infarction (MI), and ischemia-driven TLR. The cumulative estimated incidence of MACE was 3.3\%, with only one patient having a non-Q wave MI and no TLR at 1-year follow-up. No further events occurred between 1 and 5 years follow-up. ${ }^{43}$ Numerous invasive imaging modalities were used to assess the efficacy of the device, including serial (at baseline, 6 months, and 2 years) coronary angiography, IVUS, IVUS echogenicity, IVUS radiofrequency backscatter analysis, palpography and optical coherence tomography (OCT). Angiographically, the in-scaffold LLL did not differ between the 6-month and 2-year follow-up ( $0.44 \pm 0.35 \mathrm{~mm}, 0.48 \pm 0.28 \mathrm{~mm})$. Intravascular ultrasound imaging demonstrated a reduction in the lumen area between baseline and follow-up $\left(6.04 \pm 1.12 \mathrm{~mm}^{2}\right.$ at baseline versus $5.19 \pm 1.33 \mathrm{~mm}^{2}$ at 6 months and $5.47 \pm 2.11 \mathrm{~mm}^{2}$ at 2 years), which was partially attributed to the scaffold shrinkage $\left(6.94 \pm 1.70 \mathrm{~mm}^{2}\right.$ immediately postprocedure and $6.29 \pm 1.47 \mathrm{~mm}^{2}$ at the 6 months follow-up). ${ }^{44}$ Given this drawback, the scaffold was redesigned, and the new revision had a more uniform strut distribution and provided increased radial strength to vessel wall. ${ }^{45}$

The second generation of the Absorb BVS (BVS 1.1) was tested in the ABSORB Cohort B trial. A total of 101 patients (102 lesions) treated with $3.0 \mathrm{~mm} \times 18 \mathrm{~mm}$ Absorb BVS devices were enrolled in this study and divided into two groups: Cohort B1 and Cohort B2. ${ }^{46,47}$ Cohort B1 had invasive follow-up examinations (quantitative coronary angiography [QCA], IVUS, and OCT) at 6 months and 2 years, while Cohort B2 had the same investigations at 1 and 3 years. Computed tomographic coronary angiography was performed in both groups at 18 months follow-up. The rate of MACE in 101 patients was $9.0 \%$ (three non-Q-wave MI, six ischemia-driven TLR) at 2-year follow-up. ${ }^{48}$ In the Cohort B1 group, QCA analyses demonstrated an LLL of $0.19 \pm 0.18 \mathrm{~mm}$ at the 6-month, and $0.27 \pm 0.20 \mathrm{~mm}$ at the 2-year follow-up. ${ }^{49}$ IVUS examinations performed in 33 patients demonstrated the minimum lumen area to be reduced at 6 months (from $5.45 \pm 1.08 \mathrm{~mm}^{2}$ post-procedure to $\left.5.12 \pm 1.01 \mathrm{~mm}^{2}\right)$, with no changes at 2 years $\left(5.13 \pm 1.25 \mathrm{~mm}^{2}\right)$, 


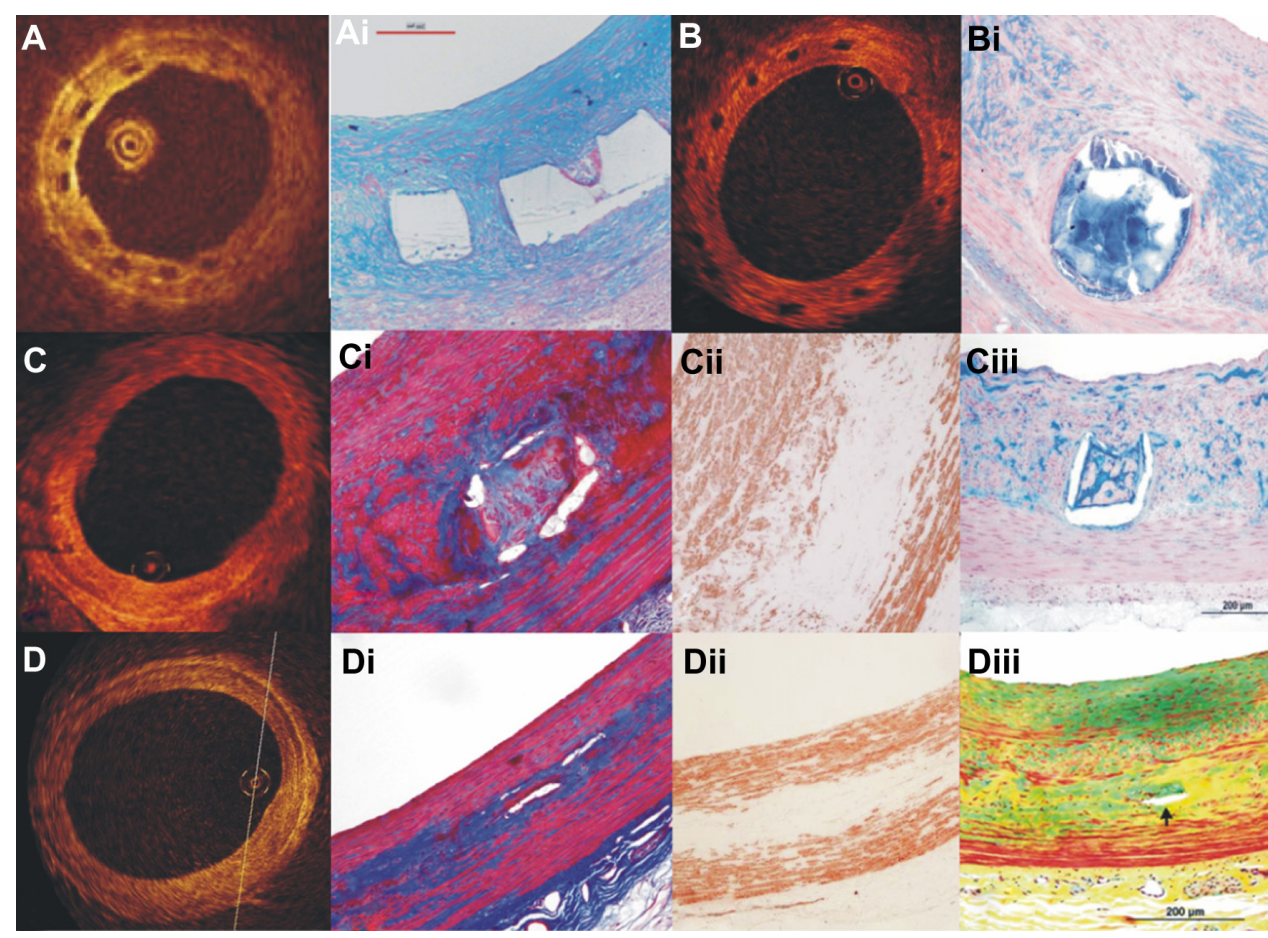

Figure 5 OCT, and corresponding histology, images at 28 days and 2, 3, and 4 years follow-up after Absorb BVS implantation. ${ }^{32}$ At 28 days, the struts appeared as preserved black boxes in OCT covered by neointimal tissue $(\mathbf{A})$; Alcian Blue Staining at 28 days confirms the structural integrity of the polymeric struts which were completely covered by neointima (Ai); at 2-year follow-up, the struts conformed to the "preserved box" in OCT (B); these "preserved boxes" structures had discrete borders and were stained positively by Alcian Blue, indicating that they were composed of acid mucopolysaccharides (proteoglycans) (Bi); at 3-year follow-up, the "preserved boxes" were only visible at 8 and 9 o'clock in OCT, $(\mathbf{C})$; at the same time point of the histological images, the trichrome staining demonstrated the presence of connective tissue in the strut footprint $(\mathbf{C i})$; the region of the preexisting strut was integrated only in a minority of cells which were observed positively with smooth muscle actin through the immunohistochemical staining (Cii); and proteoglycan matrix detected by the Alcian Blue staining (Ciii); at 4-year follow-up, the struts are no longer discernible by OCT (D); the locations in the arterial wall suggestive of prior strut location were circumscribed with connective tissue using the trichrome staining (Di); there was a paucity of cells within the connective tissue which was stained positively with smooth muscle actin (Dii); while the Movat pentachrome staining also confirmed that the strut locations were minimally discernible (Diii). Reprinted with permission Onuma Y, Serruys PW, Perkins LE, et al. Intracoronary optical coherence tomography and histology at I month and 2, 3, and 4 years after implantation of everolimus-eluting bioresorbable vascular scaffolds in a porcine coronary artery model: an attempt to decipher the human optical coherence tomography images in the ABSORB trial. Circulation. 2010;122(22):2288-2300. C Wolters Kluwer 2010.

Abbreviations: OCT, optical coherence tomography; BVS, bioresorbable vascular scaffold.

whereas the mean lumen area and mean scaffold area decreased at 6 months (from $6.53 \pm 1.24 \mathrm{~mm}^{2}$ post-procedure to $6.36 \pm 1.18 \mathrm{~mm}^{2}$ and from $6.53 \pm 1.23 \mathrm{~mm}^{2}$ post-procedure to $6.42 \pm 1.17 \mathrm{~mm}^{2}$, respectively), and then increased at 2 years $\left(6.85 \pm 1.78 \mathrm{~mm}^{2}, 7.08 \pm 1.73 \mathrm{~mm}^{2}\right.$, respectively). OCT examinations showed similar results in 23 patients. In the Cohort B2 group, the 1-year follow-up results demonstrated the LLL to be $0.27 \pm 0.32 \mathrm{~mm}$. The mean scaffold area and mean lumen area detected by IVUS were similar between post-procedure and 1 year follow-up $(6.29 \pm 0.92 \mathrm{~mm}$ versus $6.33 \pm 0.98 \mathrm{~mm}$ and $6.31 \pm 0.95 \mathrm{~mm}$ versus $6.33 \pm 1.17 \mathrm{~mm}$, respectively). OCT examinations demonstrated that the mean scaffold area did not change, whereas the mean lumen area decreased significantly by $23.4 \%(P<0.001)$.

Apart from the ongoing ABSORB Cohort B trial, three other clinical trials are underway: the ABSORB Physiology, the ABSORB II, and the ABSORB EXTEND. The ABSORB Physiology study aims to estimate the short- and longterm effects of an Absorb BVS and a Xience ${ }^{\circledR}$ (Abbott Vascular, Santa Clara, USA) stent on the physiology of the vessel wall, and will include the following metrics: vascular compliance, distensibility, endothelial responsiveness, and shear stress distribution. The ABSORB II study is the first randomized trial designed to compare the safety and efficacy of the Absorb BVS and the analogous metallic stent (Xience prime, Abbott Laboratories) in 500 patients with de novo coronary artery disease. Finally, the ABSORB EXTEND registry aims to recruit 1000 patients with de novo single or two-vessel disease and test the efficacy of the device in clinical settings. In contrast to the previous studies, this single-arm study will include long lesions and small caliber vessels with a reference vessel diameter of $2.0-2.5 \mathrm{~mm}^{50}$

\section{Magnesium metallic stents (AMSs)}

An AMS (Biotronik, Berlin, Germany) is a tubular, slotted balloon-expandable stent sculptured by laser from a tube of magnesium alloy. The device has similar mechanical strength to other metallic stents. In the first generation AMS 1.0, the resorption process was almost completed at 2 months after 
implantation, resulting in late vessel recoil. To address this limitation, the scaffold was redesigned, and in the latest revision (drug-eluting AMS, DREAM 2.0) the process was prolonged to 6 months.

The efficacy of the first generation AMS was validated in the PROGRESS-AMS (Clinical Performance and Angiographic Results of Coronary Stenting) study, which was a nonrandomized, multicenter trial that included 63 patients who had de novo lesions with lengths of 10-15 mm and reference diameters of 3.0-3.5 mm. ${ }^{51}$ During a follow-up period of 4 months, there was a $23.8 \%$ clinically driven TLR incidence, associated with a high $\operatorname{LLL}(1.08 \pm 0.49 \mathrm{~mm}) .{ }^{31}$ From 4 to 12 months, only one further patient sustained TLR, resulting in a cumulative incidence of MACE at 12 months of $26.7 \%$. These results were attributed to neointimal proliferation and vessel recoil caused by the premature reduction in the radial strength of the implanted devices. To overcome these limitations, the company added the antiproliferative drug paclitaxel, and changed the design of the scaffold and the composition of the magnesium alloy, thus providing the device with increased strength, and a prolongation of its resorption process. The efficacy of the new revision DREAMS (drug-eluting absorbable metallic stents) 1.0 was evaluated in the BIOSOLVE-1 trial. In this prospective multicenter FIM trial, 46 patients with a single de novo coronary artery lesion were implanted with 47 scaffolds. ${ }^{52}$ At 1 -year follow-up, the MACE rate was 7\% (two TLR and one MI), whereas the LLL, although improved, remained high $(0.52 \pm 0.39 \mathrm{~mm})$.

The second generation DREAMS (DREAMS 2.0) scaffold incorporates sirolimus elution instead of paclitaxel. Preclinical evaluation of the device revealed reduced inflammation, and a higher endothelization rate compared with the previous revision. Further evaluation is however needed to confirm these positive results in clinical settings and to examine its safety and efficacy in the treatment of CAD.

\section{REVA scaffold}

The REVA scaffold (Reva Medical Inc, San Diego, CA, USA) is made by a tyrosine-derived polycarbonate polymer. The first revision of the REVA scaffold had a distinctive slideand-lock mechanism that provided for a robust radial strength to the device post implantation. ${ }^{53}$

The long-term (55 months) performance of the first generation REVA scaffold was reported in a healthy porcine model recently. In this report, serial IVUS examinations showed a gradual increase in the lumen area during follow-up (lumen area change, defined as \% lumen increase post-implantation: $86 \% \pm 23 \%$ at 5 days, $94 \% \pm 10 \%$ at 12 months, and $114 \% \pm 23 \%$ at 55 months). ${ }^{54}$ The clinical performance of this first generation device was evaluated in the RESORB (The REVA endovascular study of a bioresorbable coronary stent) study. Twenty-five patients with single de novo lesions were implanted with the studied scaffolds. Angiographically, the MLD increased from $0.88 \pm 0.39 \mathrm{~mm}$ to $2.76 \pm 0.36 \mathrm{~mm}$ after device deployment, resulting in an acute gain similar to that reported with the current metallic stents. However, a high TLR rate was noted between 4- and 6-month follow-up, which was predominantly attributed to focal mechanical failures leading to the redesign of scaffold. ${ }^{55}$ The ReZolve is the second revision of REVA scaffold, has a spiral slide-and-lock mechanism, and contains the antiproliferative drug sirolimus. The new generation scaffold is currently undergoing evaluation in the RESTORE (ReZolve sirolimus-eluting bioresorbable coronary scaffold) clinical trial, which aims to investigate its safety and efficacy in 50 patients. The primary endpoints of this study are ischemic-driven TLR at 6 months, and quantitative measurements (QCA and IVUS) at 12 months. Concomitantly, a pivotal trial which aims to apply for the $\mathrm{CE}$ mark approval has been initiated.

\section{DESolve ${ }^{\mathrm{TM}}$ bioresorbable coronary scaffold}

The DESolve BRS (Elixir Medical Corporation, Sunnyvale, CA, USA) is composed of a PLLA polymer and contains two novel antiproliferative drugs (Novolimus and Myolimus). The radial strength of the device is comparable with the Elixir's BMS, and its resorption process takes approximately $2-3$ years. ${ }^{56}$

The safety and efficacy of the DESolve scaffold was evaluated in a prospective, multicenter FIM trial, which included patients with a single de novo coronary artery lesion, with a reference vessel diameter of $3.0 \mathrm{~mm}$ and lesion length $<10 \mathrm{~mm}$. At present, 16 patients have been enrolled. ${ }^{57}$ Clinically indicated TVR was reported in one patient who underwent emergency coronary artery bypass graft for a procedurally related spiral dissection at 30 days follow-up. One clinically indicated TLR occurred between 30 days and 180 days follow-up, while no cardiac death, MI, and ST have been reported at 6-month follow-up. QCA revealed an LLL of $0.19 \pm 0.19 \mathrm{~mm}$ at 6-month follow-up. IVUS examination showed a minor reduction in the lumen area and an increase in the scaffold area at 6 months $\left(5.35 \pm 0.78 \mathrm{~mm}^{2}\right.$ versus $5.10 \pm 0.78 \mathrm{~mm}^{2}$ at 6 months and $5.35 \pm 0.78 \mathrm{~mm}^{2}$ versus $5.61 \pm 0.81 \mathrm{~mm}^{2}$, respectively). OCT examination in ten patients 
showed that $98.68 \% \pm 2.44 \%$ of the struts was fully covered at 6 months follow-up, and a mean neointimal hyperplasia area of $0.71 \pm 0.36 \mathrm{~mm}^{2}$. Based on this initial promising data, the DeSolve NX study has recently commenced, and aims to evaluate the safety and efficacy of the device. The study is expected to include 120 patients with single de novo coronary artery lesions. In-scaffold LLL at 6-month follow-up is the principal endpoint. In addition, the DeSolve NX II pivotal trial has been designed to evaluate the effectiveness of the device in a broader number of patients to provide sufficient evidence for CE mark approval and is expected to start in due course. ${ }^{58}$

\section{Ideal ${ }^{\mathrm{TM}}$ BioStent}

The Ideal BioStent (Xenogenics Corp, Canton, MA, USA) is a fully bioresorbable balloon expandable scaffold. The device consists of a core backbone and a top coat. The backbone is synthesized from polylactide anhydride, and a trimer of two salicylic acid molecules joined by a sebacic acid, while the top coat part is comprised of salicylate and the antiproliferative drug sirolimus. Preclinical studies have demonstrated the potential anti-inflammatory and antiplatelet properties of salicylate, which appears to reduce restenosis and promote vessel healing during the polymer's degradation. ${ }^{59,60}$

The first generation scaffold (BTI) was examined in the "WHISPER" study. Eleven patients were included in this prospective FIM trial that evaluated the safety and efficacy of the scaffold. ${ }^{61}$ Coronary angiography and IVUS, performed post-procedurally and at follow-up, revealed the absence of scaffold recoil. However, IVUS and OCT showed increased neointimal formation, which was attributed to the inadequate drug dose and fast drug elution. In view of the high restenosis rate, the device was redesigned. The revised device (Ideal BioStent) has a higher drug dose, slower drug release kinetics, and an easy to use peel-away sheath. The new generation Ideal scaffold is currently undergoing preclinical evaluation, with a plan for the initiation of clinical trials in the near future.

\section{ART BRS}

The ART BRS (Arterial Remodeling Technologies, Noisy le Roi, France), is a fully BRS made by a PLLA amorphous polymer, without any antiproliferative drug. The new revision device was named ART18Z. The resorption process of the scaffold starts at 3 months and is expected to be completed between 18 and 24 months. To date, more than 250 devices have been implanted in porcine models, and no MACE (defined as a composite of thrombosis, death, and MI) have been reported. ${ }^{62}$ QCA analyses have shown that the
ART18Z scaffold has similar acute recoil and LLL, at 6 and 9 months, in comparison to the Multilink Vision stent (Abbott Vascular, Santa Clara, CA, USA). At 9 months follow-up, the LLL decreased in the ART18Z group, a finding that was attributed to late lumen enlargement and positive arterial remodeling. ${ }^{63}$ The performance of the ART18Z scaffold is currently being investigated in the clinical setting, in the "ARTDIVA" (Arterial Remodeling Transient DIsmantling Vascular Angioplasty) FIM trial, which commenced at five clinical centers in the third quarter of the year 2012 .

\section{Amaranth bioresorbable stent}

The Amaranth BRS (Amaranth Medical Inc, Mountain View, CA, USA) is made of PLLA, with proprietary tube fabrication. The structural integrity of the Amaranth scaffold lasts 3-6 months, and the resorption process of scaffold requires roughly $1-2$ years. Preliminary experimental studies have shown that the scaffold has a high radial strength, prolonged mechanical stability, and exhibits minimal recoil. ${ }^{64}$ A recent report evaluated the performance of the scaffold in porcine models compared with the Liberte BMS (Boston Scientific, Natick, MA, USA). A similar LLL was reported at 28- and 90-day follow-up. However, thereafter the LLL started to decrease in the Amaranth group, whereas this increased in the Liberte BMS group, from 90-day to 180-day follow-up. OCT examination at 90 days follow-up revealed a lower neointimal thickness in the Amaranth scaffold compared with the Liberte group. The company is currently designing the FIM study that will recruit 30 patients in order to evaluate the safety and efficacy of the Amaranth scaffold, with a plan for the conduction of a prospective, multicenter CE mark trial.

\section{Xinsorb BRS}

The Xinsorb BRS (Huaan Biotechnology Co, Ltd, Hangzhou, Zhejiang, People's Republic of China) is a fully bioresorbable sirolimus-loaded scaffold, consisting of poly(aspartic acid-colactide), poly( $\varepsilon$-caprolactone), and polyglycolide. The device is balloon expandable, and has radiopaque markers to facilitate its deployment. The available scaffold size ranges from 2.75 to $4.0 \mathrm{~mm}$ in diameter, and from 12 to $28 \mathrm{~mm}$ in length.

A recent experimental study evaluated the feasibility and efficacy of the Xinsorb scaffold compared with the Excel DES (JW Medical Co, Shandong, People's Republic of China). ${ }^{65}$ Sixteen Xinsorb scaffolds and 16 Excel stents were implanted in the coronary arteries of porcine models. ${ }^{66}$ Histomorphometry demonstrated a lower percentage diameter restenosis in the Xinsorb scaffold compared with 
the Excel DES (at 30-day follow-up, 18.6\% $\pm 5.2 \%$ versus $21.4 \% \pm 7.2 \%, P>0.05$; at 90 -day follow-up, $24.5 \% \pm 4.7 \%$ versus $27.7 \% \pm 5.6 \%, P>0.05$, respectively). Although these preliminary results are encouraging, further extensive preclinical studies are necessary to investigate the safety and efficacy of the scaffold. The company is expected to organize an FIM trial in 2013.

\section{Acute BRS}

The Acute BRS (OrbusNeich, Fort Lauderdale, FL, USA) is a tube-shaped lockable and balloon-expandable BRS. The device consists of three polymeric materials (poly-L-lactideco- $\varepsilon$-caprolactone, PDLA, and PLLA) and incorporates a partitioned coating technology, which allows for the scaffold to be covered by abluminal sirolimus and luminal endothelial progenitor cell capture (+CD34) antibodies. Preliminary preclinical evaluation demonstrated an optimal device implantation without evidence of fracture. ${ }^{67}$ Updated data are expected to be presented in the EuroPCR Focus Group 2013.

\section{MeRes ${ }^{\mathrm{TM}}$ BRS}

MeRes BRS (Meril Life Sciences, Vapi, Gujarat, India) is a merilimus eluting bioresorbable coronary scaffold. The device is comprised of a new PLA formulation and has a hybrid scaffold geometry structure, which gives the scaffold a high radial strength. The performance of the device was examined in an experimental animal study. Initial results showed that the elution of the coated drug merilimus lasts more than 30 days, with no evidence of inflammatory reaction during the polymer's biodegradation. ${ }^{64}$

\section{FADES BRS}

The FADES scaffold (Zorion Medical, Indianapolis, IN, USA) is a fully bioresorbable drug-eluting scaffold. The polymer of the scaffold involves a hybrid material of magnesium alloy that includes rare earth elements and PLGA. Preclinical studies showed that the device was completely absorbed with little to no inflammatory tissue response within 90 days. ${ }^{64}$ Further preclinical evaluations are needed before the conduction of the FIM study.

\section{Other BRSs}

Three other devices are under development: the Avatar BRS (S3V Vascular Technologies, Bangalore, Karnataka, India), the Sahajanand BRS (Sahajanand Medical Technologies, Surat, Gujarat, India), and Stanza ${ }^{\mathrm{TM}}$ BRS (480 Biomedical, Boston, Massachusetts, USA). All these three BRSs are in their infancy, and preclinical and clinical evaluation is pending. ${ }^{64}$

\section{Conclusion}

BRSs introduce a unique potential in the treatment of coronary lesions, as they provide temporary vessel scaffolding, and then they disappear, thereby allowing for the restoration of the vessel wall physiology and vasomotion. Initial preclinical and clinical results appear promising. However, it should be mentioned that our clinical data are limited to simple lesions and there is no evidence in the context of randomized control trials that would allow direct comparison of the efficacy of the BRS with the effectiveness of the new generation DES. Furthermore, BRS technology has significant limitations such as the bulky nature of the devices, the fact that they cannot be implemented to treat bifurcation lesions requiring a two stent strategy, the concerns about an increased risk of restenosis in case of overlapping, and their limited extensibility and the risk of scaffold fracture that limit their applications in the current clinical practice. Therefore further development is necessary so as to reduce the thickness of the struts and increase the extensibility of the devices so as to overcome the current pitfalls of BRS technology and become the workhorse device for the treatment of CAD.

\section{Disclosure}

The authors have no conflict of interest and did not receive grants or financial support from industry or from any other source to prepare this manuscript.

\section{References}

1. Gruntzig A. Transluminal dilatation of coronary-artery stenosis. Lancet. 1978;1(8058):263.

2. Gruntzig AR, Senning A, Siegenthaler WE. Nonoperative dilatation of coronary-artery stenosis: percutaneous transluminal coronary angioplasty. N Engl J Med. 1979;301(2):61-68.

3. Gruentzig AR, King SB 3rd, Schlumpf M, Siegenthaler W. Long-term follow-up after percutaneous transluminal coronary angioplasty. The early Zurich experience. N Engl J Med. 1987;316(18):1127-1132.

4. Nobuyoshi M, Kimura T, Nosaka H, et al. Restenosis after successful percutaneous transluminal coronary angioplasty: serial angiographic follow-up of 229 patients. J Am Coll Cardiol. 1988;12(3):616-623.

5. OrmistonJA, StewartFM, RocheAH, WebberBJ, Whitlock RM, WebsterMW. Late regression of the dilated site after coronary angioplasty: a 5-year quantitative angiographic study. Circulation. 1997;96(2):468-474.

6. Fischman DL, Leon MB, Baim DS, et al. A randomized comparison of coronary-stent placement and balloon angioplasty in the treatment of coronary artery disease. Stent Restenosis Study Investigators. $N$ Engl J Med. 1994;331(8):496-501.

7. Mintz GS, Popma JJ, Pichard AD, et al. Arterial remodeling after coronary angioplasty: a serial intravascular ultrasound study. Circulation. 1996;94(1):35-43.

8. Moses JW, Leon MB, Popma JJ, et al. Sirolimus-eluting stents versus standard stents in patients with stenosis in a native coronary artery. N Engl J Med. 2003;349(14):1315-1323. 
9. Serruys PW, de Jaegere P, Kiemeneij F, et al. A comparison of balloon-expandable-stent implantation with balloon angioplasty in patients with coronary artery disease. Benestent Study Group. $N$ Engl J Med. 1994;331(8):489-495.

10. Stone GW, Ellis SG, Cox DA, et al. A polymer-based, paclitaxel-eluting stent in patients with coronary artery disease. N Engl J Med. 2004; 350(3):221-231.

11. Farooq V, Gogas BD, Serruys PW. Restenosis: delineating the numerous causes of drug-eluting stent restenosis. Circ Cardiovasc Interv. 2011;4(2):195-205.

12. Serruys PW, Silber S, Garg S, et al. Comparison of zotarolimus-eluting and everolimus-eluting coronary stents. $N$ Engl J Med. 2010;363(2): 136-146.

13. Stone GW, Moses JW, Ellis SG, et al. Safety and efficacy of sirolimus- and paclitaxel-eluting coronary stents. $N$ Engl J Med. 2007; 356(10):998-1008.

14. Windecker S, Remondino A, Eberli FR, et al. Sirolimus-eluting and paclitaxel-eluting stents for coronary revascularization. $N$ Engl J Med. 2005;353(7):653-662.

15. Windecker S, Serruys PW, Wandel S, et al. Biolimus-eluting stent with biodegradable polymer versus sirolimus-eluting stent with durable polymer for coronary revascularisation (LEADERS): a randomised non-inferiority trial. Lancet. 2008;372(9644):1163-1173.

16. Zhang Y, Farooq V, Garcia-Garcia HM, et al. Comparison of intravascular ultrasound versus angiography-guided drug-eluting stent implantation: a meta-analysis of one randomised trial and ten observational studies involving 19,619 patients. Euro Intervention. 2012;8(7):855-865.

17. Baber U, Mehran R, Sharma SK, et al. Impact of the everolimus-eluting stent on stent thrombosis: a meta-analysis of 13 randomized trials. $\mathrm{JAm}$ Coll Cardiol. 2011;58(15):1569-1577.

18. McFadden EP, Stabile E, Regar E, et al. Late thrombosis in drug-eluting coronary stents after discontinuation of antiplatelet therapy. Lancet. 2004;364(9444):1519-1521

19. Serruys PW, Ormiston JA, Onuma Y, et al. A bioabsorbable everolimus-eluting coronary stent system (ABSORB): 2-year outcomes and results from multiple imaging methods. Lancet. 2009;373(9667): 897-910.

20. Stefanini GG, Kalesan B, Serruys PW, et al. Long-term clinical outcomes of biodegradable polymer biolimus-eluting stents versus durable polymer sirolimus-eluting stents in patients with coronary artery disease (LEADERS): 4 year follow-up of a randomised non-inferiority trial. Lancet. 2011;378(9807):1940-1948.

21. Bourantas CV, Onuma Y, Farooq V, Zhang Y, Garcia-Garcia HM, Serruys PW. Bioresorbable scaffolds: current knowledge, potentialities and limitations experienced during their first clinical applications. Int $J$ Cardiol. Epub June 28, 2012.

22. Bourantas CV, Zhang Y, Farooq V, Garcia-Garcia HM, Onuma Y, Serruys PW. Bioresorbable scaffolds: current evidence and ongoing clinical trials. Curr Cardiol Rep. 2012;14(5):626-634.

23. Finn AV, Joner M, Nakazawa G, et al. Pathological correlates of late drug-eluting stent thrombosis: strut coverage as a marker of endothelialization. Circulation. 2007;115(18):2435-2441.

24. Kotani J, Awata M, Nanto S, et al. Incomplete neointimal coverage of sirolimus-eluting stents: angioscopic findings. $\mathrm{J} \mathrm{Am} \mathrm{Coll} \mathrm{Cardiol}$. 2006;47(10):2108-2111.

25. Serruys PW, Garcia-Garcia HM, OnumaY. From metallic cages to transient bioresorbable scaffolds: change in paradigm of coronary revascularization in the upcoming decade. Eur Heart J. 2012;33(1):16b-25b.

26. Onuma Y, Serruys PW. Bioresorbable scaffold: the advent of a new era in percutaneous coronary and peripheral revascularization. Circulation. 2011;123(7):779-797.

27. van der Giessen WJ, Slager CJ, van Beusekom HM, et al. Development of a polymer endovascular prosthesis and its implantation in porcine arteries. J Interv Cardiol. 1992;5(3):175-185.

28. van der Giessen WJ, Lincoff AM, Schwartz RS, et al. Marked inflammatory sequelae to implantation of biodegradable and nonbiodegradable polymers in porcine coronary arteries. Circulation. 1996;94(7):1690-1697.
29. Lincoff AM, Furst JG, Ellis SG, Tuch RJ, Topol EJ. Sustained local delivery of dexamethasone by a novel intravascular eluting stent to prevent restenosis in the porcine coronary injury model. $\mathrm{J} \mathrm{Am} \mathrm{Coll}$ Cardiol. 1997;29(4):808-816.

30. Yamawaki T, Shimokawa H, Kozai T, et al. Intramural delivery of a specific tyrosine kinase inhibitor with biodegradable stent suppresses the restenotic changes of the coronary artery in pigs in vivo. $\mathrm{J} \mathrm{Am} \mathrm{Coll}$ Cardiol. 1998;32(3):780-786.

31. Erbel R, Di Mario C, Bartunek J, et al. Temporary scaffolding of coronary arteries with bioabsorbable magnesium stents: a prospective, non-randomised multicentre trial. Lancet. 2007;369(9576):1869-1875.

32. Onuma Y, Serruys PW, Perkins LE, et al. Intracoronary optical coherence tomography and histology at 1 month and 2, 3, and 4 years after implantation of everolimus-eluting bioresorbable vascular scaffolds in a porcine coronary artery model: an attempt to decipher the human optical coherence tomography images in the ABSORB trial. Circulation. 2010;122(22):2288-2300

33. Ormiston JA, Serruys PW, Regar E, et al. A bioabsorbable everolimus-eluting coronary stent system for patients with single de-novo coronary artery lesions (ABSORB): a prospective open-label trial. Lancet. 2008;371(9616):899-907.

34. English JP, PDE. Polyglycolide and Polylactide. In: Wiseman DM, Kost J, Domb AJ, editors. Handbook of Biodegradable Polymers. Boca Raton, FL: CRC Press; 1998.

35. Kronenthal R. Biodegradable Polymers in Medicine and Surgery. In: Kronenthal R, Oser Z, Martin E, editors.Polymers in Medicine and Surgery. New York, NY: Plenum Press; 1975:119-137.

36. Garg S, Serruys P. Biodegradable stents and non-biodegradable stents. Minerva Cardioangiol. 2009;57(5):537-565.

37. Ormiston JA, Serruys PW. Bioabsorbable coronary stents. Circ Cardiovasc Interv. 2009;2(3):255-260.

38. Waksman R. Lessons learnt from preclinical studies of magnesium scaffolds (Biotronik's DREAMS program). EuroPCR; Mar 2012; Rotterdam, The Netherlands.

39. Tamai H, Igaki K, Kyo E, et al. Initial and 6-month results of biodegradable poly-1-lactic acid coronary stents in humans. Circulation. 2000;102(4):399-404.

40. Nishio S, Kosuga K, Igaki K, et al. Long-term ( $>10$ years) clinical outcomes of first-in-human biodegradable poly-1-lactic acid coronary stents: Igaki-Tamai stents. Circulation. 2012;125(19):2343-2353.

41. Onuma Y, Garg S, Okamura T, et al. Ten-year follow-up of the IGAKI-TAMAI stent. A posthumous tribute to the scientific work of Dr Hideo Tamai. Euro Intervention. 2009;5 Suppl F:F109-F111.

42. Onuma Y, Serruys PW, Perkins LE, et al. Intracoronary optical coherence tomography and histology at 1 month and 2, 3, and 4 years after implantation of everolimus-eluting bioresorbable vascular scaffolds in a porcine coronary artery model: an attempt to decipher the human optical coherence tomography images in the ABSORB trial. Circulation. 2010;122(22):2288-2300.

43. Serruys PW, Ormiston JA, OnumaY, etal. A bioabsorbable everolimus-eluting coronary stent system (ABSORB): 2-year outcomes and results from multiple imaging methods. Lancet. 2009;373(9667): 897-910.

44. Tanimoto S, Bruining N, van Domburg RT, et al. Late stent recoil of the bioabsorbable everolimus-eluting coronary stent and its relationship with plaque morphology. J Am Coll Cardiol. 2008;52(20):1616-1620.

45. Tanimoto S, Serruys PW, Thuesen L, et al. Comparison of in vivo acute stent recoil between the bioabsorbable everolimus-eluting coronary stent and the everolimus-eluting cobalt chromium coronary stent: insights from the ABSORB and SPIRIT trials. Catheter Cardiovasc Interv. 2007;70(4):515-523.

46. Diletti R, Onuma Y, Farooq V, et al. 6-month clinical outcomes following implantation of the bioresorbable everolimus-eluting vascular scaffold in vessels smaller or larger than $2.5 \mathrm{~mm}$. J Am Coll Cardiol. 2011;58(3):258-264.

47. Serruys PW, Onuma Y, Dudek D, et al. Evaluation of the second generation of a bioresorbable everolimus-eluting vascular scaffold for the treatment of de novo coronary artery stenosis: 12-month clinical and imaging outcomes. J Am Coll Cardiol. 2011;58(15):1578-1588. 
48. Ormiston JA, Serruys PW, Onuma Y, et al. First serial assessment at 6 months and 2 years of the second generation of absorb everolimuseluting bioresorbable vascular scaffold: a multi-imaging modality study. Circ Cardiovasc Interv. 2012;5(5):620-632.

49. Serruys PW, Onuma Y. Vascular reparative therapy - a dream coming true? EuroPCR; May 16, 2012; Rotterdam, The Netherlands.

50. Farooq V, Gomez-Lara J, Brugaletta S, et al. Proximal and distal maximal luminal diameters as a guide to appropriate deployment of the ABSORB everolimus-eluting bioresorbable vascular scaffold: a sub-study of the ABSORB Cohort B and the on-going ABSORB EXTEND Single Arm Study. Catheter Cardiovasc Interv. 2012;79(6):880-888.

51. Ghimire G, Spiro J, Kharbanda R, et al. Initial evidence for the return of coronary vasoreactivity following the absorption of bioabsorbable magnesium alloy coronary stents. Euro Intervention. 2009;4(4):481-484.

52. Waksman R. PROGRESS was made toward DREAM: magnesium Stent. EuroPCR; May 17, 2012; Rotterdam, The Netherlands.

53. Pollman MJ. Engineering a bioresorbable stent: REVA programme update. Euro Intervention. 2009;5 Suppl F:F54-F57.

54. Strandberg E, Zeltinger J, Schulz DG, Kaluza GL. Late positive remodeling and late lumen gain contribute to vascular restoration by a non-drug eluting bioresorbable scaffold: a four-year intravascular ultrasound study in normal porcine coronary arteries. Circ Cardiovasc Interv. 2012;5(1):39-46.

55. Grube E. The Boston scientific and REVA technology. EuroPCR; May 22, 2009; Barcelona, Spain.

56. Yan J, Bhat VD. Elixir Medical's bioresorbable drug eluting stent (BDES) programme: an overview. Euro Intervention. 2009; 5 Suppl F:F80-F82.

57. Verheye S. DESolve first in man study - preliminary results. EuroPCR; Mar 2012; Rotterdam, The Netherlands.
58. Verheye S. DESolve myolimus eluting bioresorbable coronary scaffold first in man trial - imaging and clinical results at 6 months. EuroPCR; May 16, 2012; Rotterdam, The Netherlands.

59. Jabara R, Chronos N, Robinson K. Novel bioabsorbable salicylate-based polymer as a drug-eluting stent coating. Catheter Cardiovasc Interv. 2008;72(2):186-194.

60. Jabara R, Pendyala L, Geva S, Chen J, Chronos N, Robinson K. Novel fully bioabsorbable salicylate-based sirolimus-eluting stent. Euro Intervention. 2009;5 Suppl F:F58-F64.

61. Jabara R. Poly-anhydride based on salicylic acid and adipic acid anhydride. EuroPCR; May 22, 2009; Barcelona, Spain.

62. Lafont A, Durand E. ART: concept of a bioresorbable stent without drug elution. Euro Intervention. 2009;5 Suppl F:F83-F87.

63. Lafont A. ART bioresorbable scaffold dedicated for vascular scaffolding. EuroPCR; May 17, 2012.

64. cvPIPELINE [database on the Internet]. Santa Barbara, CA: MarketMonitors Inc. Available from: http://www.cvpipeline.com. Accessed September 20, 2012.

65. Han Y, Zhang L, Yang L, et al. A new generation biodegradable polymercoated sirolimus-eluting stents for the treatment of coronary artery disease: final 5-year clinical outcomes from the CREATE study. Euro Intervention. 2012;8(7):815-822.

66. Shen L, Wand Qi-bing, Wu Yi-zhe, et al. Preliminary evaluation of fully bioabsorbable PLLA sirolimus eluting stents in a porcine model. Chin J Intervent Cardiol. 2009;19(6):301-305.

67. Cottone RJ, Thatcher GL, Parker SP, et al. OrbusNeich fully absorbable coronary stent platform incorporating dual partitioned coatings. Euro Intervention. 2009;5 Suppl F:F65-F71.
Medical Devices: Evidence and Research

\section{Publish your work in this journal}

Medical Devices: Evidence and Research is an international, peerreviewed, open access journal that focuses on the evidence, technology, research, and expert opinion supporting the use and application of medical devices in the diagnosis, treatment and management of clinical conditions and physiological processes. The identification of novel

\section{Dovepress}

devices and optimal use of existing devices which will lead to improved clinical outcomes and more effective patient management and safety is a key feature. The manuscript management system is completely online and includes a quick and fair peer-review system. Visit http://www. dovepress.com/testimonials.php to read real quotes from authors. 